\title{
Desarrollo e implementación de una veleta de corte a alta revolución para sondajes
}

\section{Development and performance of a shear vane at high revolution in boreholes}

Fecha de entrega: 30 de diciembre 2014 Fecha de aceptación: 22 de abril 2015

\section{Roberto Olguín y Mauricio Ortúzar}

IDIEM, Universidad de Chile, Plaza Ercilla 883, Santiago, Chile, roberto.olguin@idiem.cl, mauricio.ortuzar@idiem.cl

El ensayo de veleta de corte es un método de prospección in situ clásico. Desde su origen, la metodología estandarizada de este ensayo se ha orientado a determinar la resistencia al corte no drenada $S_{u}$, de suelos finos de baja permeabilidad. Considerando la necesidad de la Ingeniería Geotécnica por caracterizar suelos arenosos finos y/o suelos finos de mayor permeabilidad, en este trabajo se presenta el desarrollo e implementación de la veleta de corte adaptada para alcanzar velocidades de giro en torno a las $100 \mathrm{rpm}$. Dicha velocidad de rotación permite asegurar una respuesta no drenada en materiales del tipo arenas finas limosas. Asimismo, este ensayo se desarrolló para ser implementado en máquinas de sondaje geotécnico, lo que permite la ejecución de la veleta a diversas profundidades. Se presentan resultados obtenidos en lamas de relave. El documento presenta la composición del equipo desarrollado -partes mecánicas y sistema de adquisición de datos, metodología de ensayo, resultados obtenidos en pruebas iniciales y comentarios finales.

Palabras clave: veleta de corte, resistencia al corte no drenada, caracterización geotécnica.
The vane shear test is a traditional in situ method. From its origin, the standardized methodology of this test has been orientated to obtain the undrained shear strength $S_{u}$ of fine grained soils with low permeability. Considering the geotechnical need for characterizing fine sandy soils and/or fine grained soils with higher permeability, this article presents the development and implementation of a vane shear test adapted to reach angular velocities of around $100 \mathrm{rpm}$. This rotational velocity ensures an undrained behaviour on materials such as silty fine sands. Also, this test was developed to be implemented on drilling rigs in order to make vane tests at different depths. Results obtained in tailings are presented. In this article, the composition of the equipment developed - mechanical parts and data acquisition system- test methodology, initial test results and final comments are presented.

Keywords: vane shear test, undrained shear strength, geotechnical characterization

\section{Introducción}

El desarrollo e implementación de la veleta de corte como ensayo in-situ para la estimación de la resistencia no drenada de suelos finos se remonta a los años 1940 a 1950 (Ladd et al., 1977), siendo uno de los métodos de prospección más clásicos y de mayor uso por ingenieros geotécnicos. Con el correr de los años su aplicación se ha ido estandarizando en parámetros tales como dimensiones, relación diámetro con altura y velocidad de rotación, aunque siempre bajo el contexto de ser usado en suelos finos debido a que la baja permeabilidad de estos materiales genera que el suelo responda de forma no drenada frente a la movilización de la falla en relación a las velocidades de rotación definidas y normadas.

Por otra parte, la estimación de la resistencia no drenada en lamas de relave ha sido más recurrente a partir de los diversos proyectos de crecimiento de los tranques o bien como input en los análisis de estabilidad de depósitos en los cuales se proyecta la colocación de relaves espesados sobre las mencionadas lamas. En este sentido, los métodos de uso común en Chile corresponden a triaxiales CIU en muestras inalteradas obtenidas mediante tubo muestreador 
Shelby o extraídas de calicatas, simulación de la depositación a través del método de confección de probeta denominado slurry para posteriormente efectuar ensayos triaxiales sobre estas probetas, y ensayos de terreno SPT (Standard Penetration Test) en sondajes y, últimamente, penetraciones CPT-u. Naturalmente, cada método contiene ventajas y desventajas, siendo materia de análisis de los especialistas en Mecánica de Suelos la confiabilidad en la estimación de la resistencia no drenada de cada uno de los métodos mencionados.

De esta forma, desarrollar un equipo de veleta de corte que permita en primer lugar generar una superficie de falla cilíndrica en lamas a una velocidad tal que se asegure una respuesta no drenada del suelo, y en segundo lugar que este ensayo pueda realizarse ha profundidades de interés para el especialista, ha sido la motivación para la implementación del equipo que se presenta en este artículo, cuyo objetivo principal es el de proporcionar a los ingenieros geotécnicos una nueva herramienta de ensayo in situ para la estimación de la resistencia no drenada en lamas de relave u otros materiales de similar granulometría y permeabilidad. Junto a la descripción de los componentes que conforman el equipo, se presentan algunos resultados obtenidos en lamas de relave.

\section{Breve reseña de la veleta de corte}

La veleta de corte consiste en una cruceta metálica de dimensiones estandarizadas que se inserta en el suelo a analizar. Una vez colocada, se aplica un torque sobre el sistema de barras con el fin de movilizar una superficie de falla cilíndrica, tal como se muestra en Figura 1. Al rotar la veleta, $\mathrm{y}$ tras alcanzar un torque máximo, el procedimiento indicado tanto en el Eurocode (2000) como en ASTM D 2573 (2008) sugieren realizar 10 vueltas adicionales de manera de lograr un estado de suelo remoldeado o residual, registrando también el torque asociado a esta condición (torque residual).

Para veletas de corte cuyas láminas son rectangulares (también existen veletas con láminas que terminan con puntas recortadas) y cuya relación alto:diámetro es $2: 1$, es posible demostrar que la resistencia al corte no drenada queda dada por la siguiente ecuación (Wroth, 1984; Powrie, 2004):

$$
S_{\mathrm{u}}=0.273 \frac{T}{D^{3}}
$$

donde $S_{\mathrm{u}}$ es la resistencia al corte no drenada, $T$ es el torque aplicado a la veleta y $D$ es el diámetro de la veleta. Para el cálculo de (1), se asume que tanto el manto cilíndrico como ambos círculos de los extremos (superior e inferior) generan resistencia. Al respecto, si bien considerar la totalidad del área superior es discutible, esta hipótesis es conservadora en el cálculo de $S_{\mathrm{u}}$ respecto a no considerarla. En caso de utilizar el torque máximo del ensayo, se obtendrá el valor de $S_{\mathrm{u}}$ máximo. Asimismo, si se utiliza el torque residual o remoldeado, se obtendrá el correspondiente valor residual de $S_{\mathrm{u}}$. Al respecto, la Figura 2 presenta una curva torque-ángulo de giro de un ensayo de veleta en suelo inalterado.

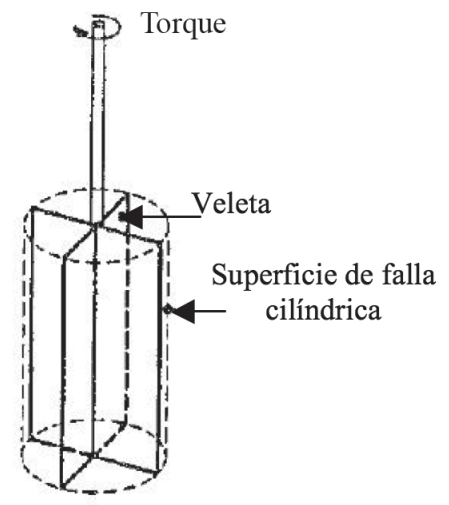

Figura 1: Esquema veleta de corte y superficie de falla

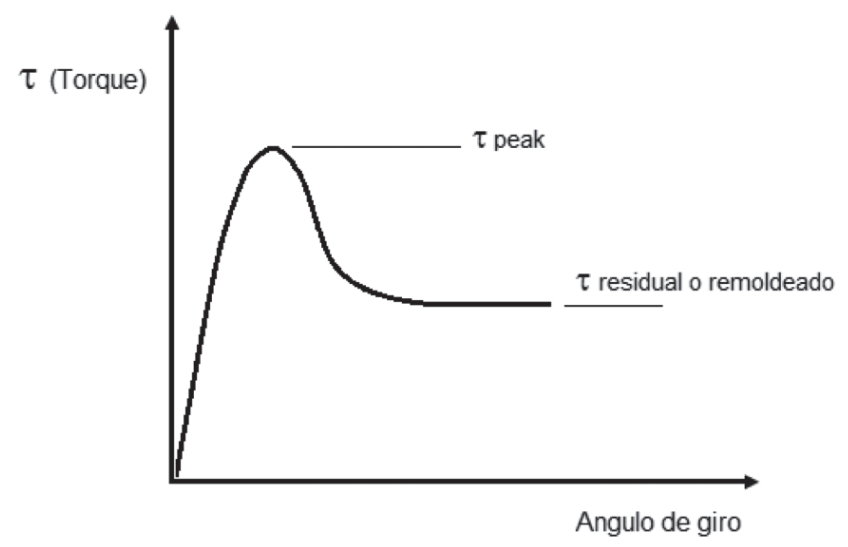

Figura 2: Resultado tipo torque-ángulo de veleta de corte en suelo inalterado

Respecto a la velocidad de rotación, y considerando que la veleta de corte es utilizada en suelos finos cohesivos, el Eurocode (2000) sugiere rotaciones de entre 0.1 y $0.2 \%$ ( 0.017 y 0.033 rpm respectivamente), mientras que laASTM 
D 2573 (2008) indica que la velocidad de rotación debe ser igual a $0.1 \% \mathrm{~s}(0.017 \mathrm{rpm})$. Al tener suelos más permeables en relación con los suelos finos cohesivos, como lo son las lamas de relave, estas velocidades de rotación no permiten asegurar que el giro de la veleta generará respuesta no drenada en el suelo, sino que probablemente los excesos de presión de poros se alcanzan a disipar. En consecuencia, y con el fin soslayar este efecto, el equipo desarrollado permite generar velocidades angulares de ensayo en torno a $100 \mathrm{rpm}$, velocidad que se estima suficiente para generar una respuesta no drenada. En este sentido, cabe señalar que lo presentado en este artículo corresponde a las primeras experiencias desarrolladas en IDIEM en relación a veletas de alta revolución, por lo que podría darse el caso que futuras pruebas, en las cuales se realicen ensayos variando la velocidad angular, proporcionen mayores antecedentes respecto a la definición de la mencionada velocidad de giro.

\section{Composición del equipo}

El equipo de veleta de corte fue desarrollado para acoplarse al sistema de rotación de la sonda de IDIEM modelo Longyear LG-520 (ver Figura 3). Esta sonda geotécnica dispone de un sistema que permite regular la velocidad de rotación de las barras, lo que fue de gran utilidad en la etapa de las pruebas iniciales del sistema, testeando rotaciones entre 85 y $200 \mathrm{rpm}$.

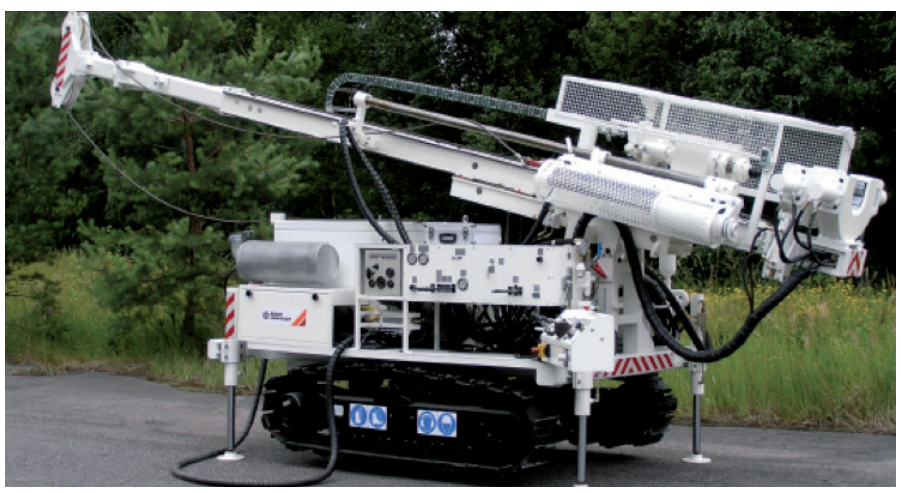

Figura 3: Sonda geotécnica LG-520

En adición a la sonda, los principales equipos utilizados en la implementación de la veleta son:

- Sensores de torque electrónicos, de $100 \mathrm{Nm}$ y 1000 $\mathrm{Nm}$ de capacidad máxima y precisión del $1 \%$ de esta capacidad (Figura 4).
- Procesador de datos ARM CORTEX 32 bits, con una tasa de muestreo de 2000 datos por segundo.

- Barras BQ que conforman el sistema de rotación de la veleta.

- Barras HQ utilizadas como revestimiento del pozo.

- Rodamientos de alto rendimiento.

- Acoples y conexiones para incorporación del sensor de torque y la veleta al sistema de rotación (Figura 5).

- Veleta metálica de $55 \mathrm{~mm}$ de diámetro, $110 \mathrm{~mm}$ de alto, $2 \mathrm{~mm}$ de espesor de las platinas o aspas, vástago de 12.7 $\mathrm{mm}$ de diámetro y $55 \mathrm{~cm}$ de largo (Figuras 6 y 7).

- Notebook para almacenamiento y procesamiento de datos.

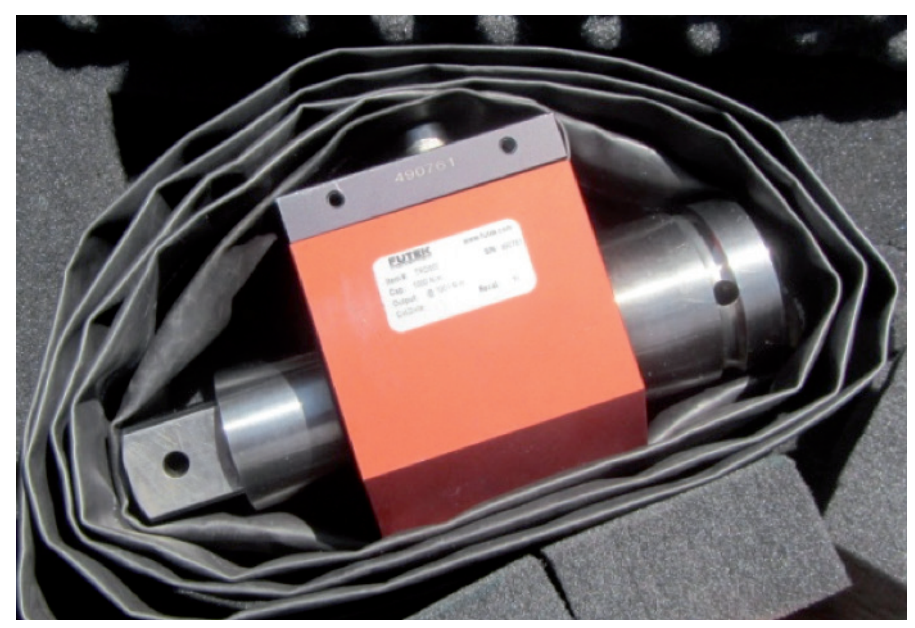

Figura 4: Torquímetro eléctrico

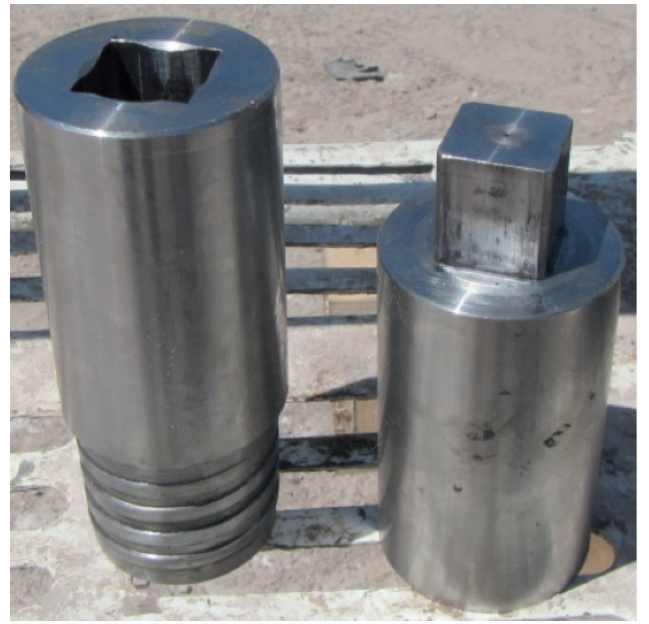

Figura 5: Acoples con dado para conexión macho-hembra a torquímetro 

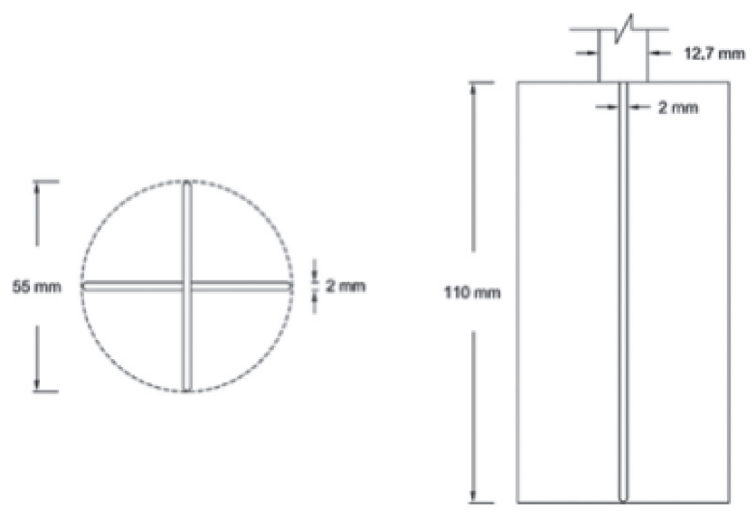

Figura 6: Dimensiones veleta de corte

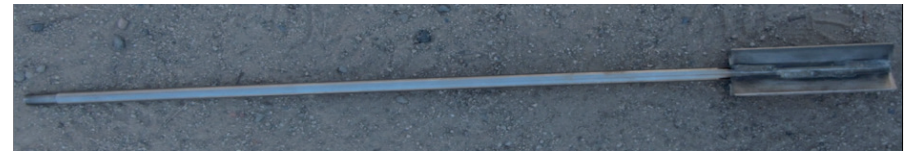

Figura 7: Veleta de corte
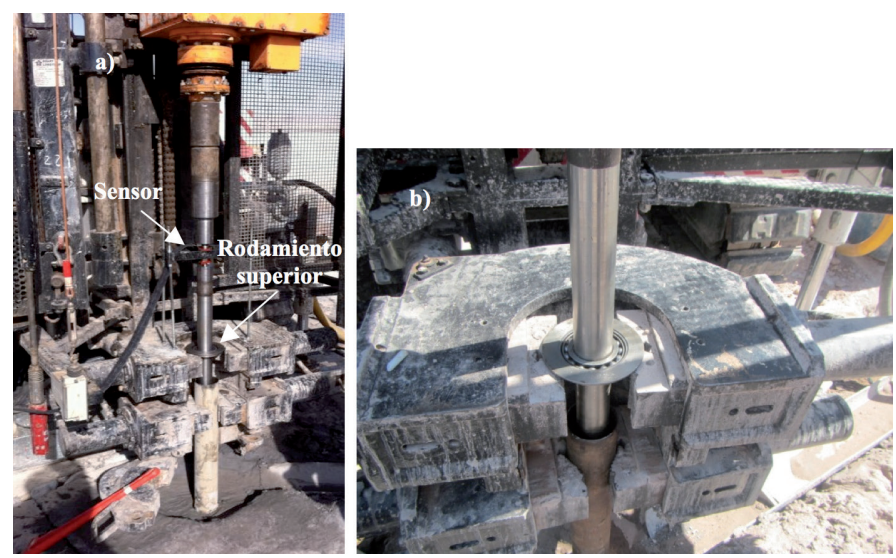

Figura 8: a) Rodamiento en sistema montado y b) acercamiento rodamiento superior

El sensor eléctrico de torque se incorpora al eje de rotación en la parte superior del sistema de barras (ver Figura 8) mediante la conexión de acoples macho-hembra rectangulares como los mostrados en la Figura 5. Este sensor, que mide torque y ángulo de giro, se conecta a la caja registradora y procesadora de datos para su operación por parte del ingeniero especialista. Además, con el objetivo de mantener la verticalidad del sistema y reducir el roce (y por lo tanto los torques parásitos), se colocan dos rodamientos de alto rendimiento, uno bajo el sensor de torque (ver Figura 8, rodamiento superior) y el otro en la salida inferior, en donde se conecta la veleta (Figura 9). Además, para reducir el pandeo de las barras BQ, se colocan rodamientos entre las uniones de éstas, los cuales se apoyan por el interior de la barra de revestimiento de diámetro HQ. El esquema del equipo de veleta instalado se presenta en Figura 10.

Como se aprecia en la Figura 8b, una mordaza sujeta el revestimiento HQ mientras que la otra fija el rodamiento superior por donde rota el sistema de barras BQ.

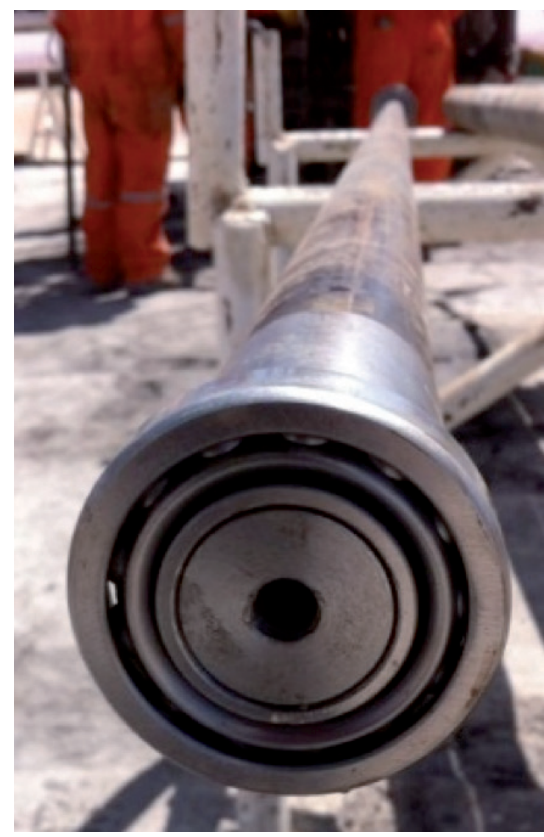

Figura 9: Rodamiento inferior, con sistema para conexión de veleta

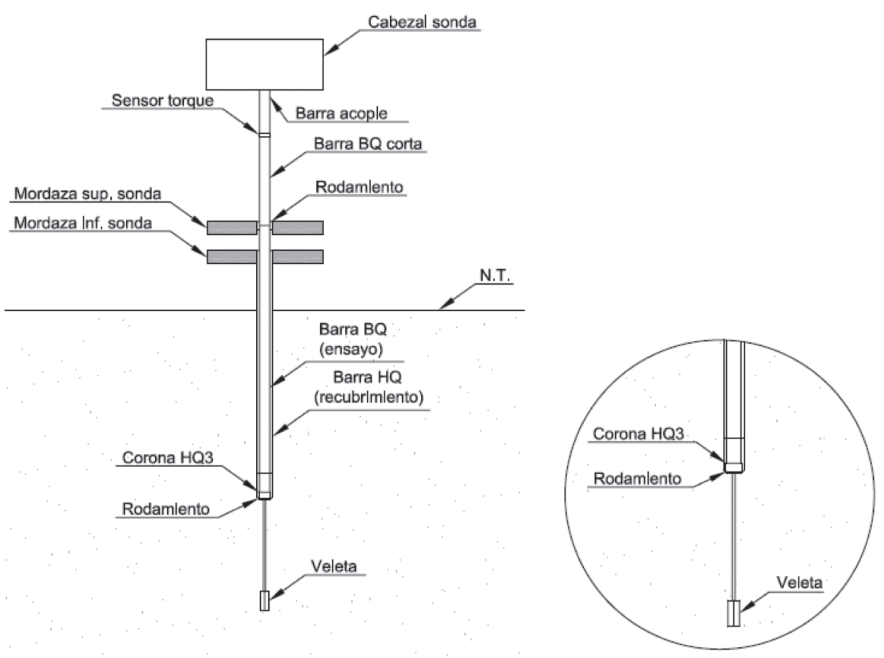

Figura 10: Esquema del equipo instalado para ensayo.

\section{Metodología de ensayo}

Instalada la sonda geotécnica en el punto de perforación, en primera instancia se perfora con corona diamantina 
HQ3 hasta alcanzar una profundidad igual a $55 \mathrm{~cm}$ por sobre la cota de ensayo de veleta que se ha especificado. De esta forma, todo el sistema de barras de diámetro HQ queda revistiendo el pozo. Luego, se introduce el sistema de barras BQ y se conecta el torquímetro y rodamientos, sin la colocación de la veleta. Una vez instalado, se realizan al menos cinco pruebas iniciales, esto para medir los torques parásitos inherentes al sistema de barras BQ, rodamientos, acoples y efectos de turbulencia por el agua que gira en la salida del rodamiento, medición que posteriormente permite corregir los resultados de la rotación con la veleta instalada.

Finalizado el proceso de calibración, se retiran las barras BQ para instalar la veleta en el extremo inferior, para luego volver a introducirlas por el interior de las barras HQ. Una vez alcanzado el suelo natural, la veleta es insertada mediante la presión continua que proporciona la sonda (no se utiliza golpeteo o rotación) en forma análoga a la metodología utilizada para muestreo con tubo de pared delgada Shelby. Tras el posicionamiento de la veleta, se conecta el sistema electrónico de recolección de datos y se realiza el ensayo aplicando una velocidad de rotación en torno a las $100 \mathrm{rpm}$.

\section{Pruebas iniciales}

Durante el transcurso de la implementación del ensayo de veleta de corte a altas revoluciones, se realizaron ensayos de rotación sin veleta para visualizar los efectos tanto del sistema de barras como de la metodología de ensayo en la medición del torque. Para esto, se realizaron rotaciones a diferentes velocidades angulares en un sondaje de prueba, instalando $15 \mathrm{~m}$ de barras BQ. Se utilizó el torquímetro de capacidad máxima de $100 \mathrm{Nm}$. Los resultados obtenidos se presentan en la Figura 11.

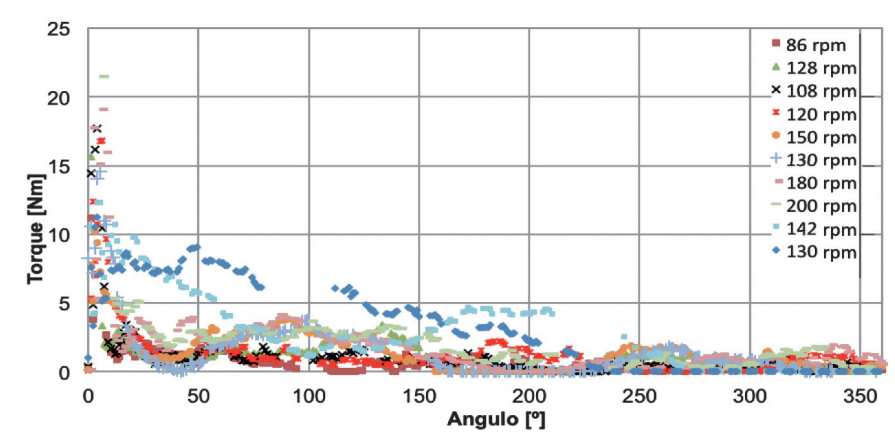

Figura 11: Pruebas iniciales "al aire", con $15 \mathrm{~m}$ de barras BQ
Los resultados muestran que al inicio de la rotación se generan torques con valores entre 10 y $21 \mathrm{Nm}$ aproximadamente, coincidiendo que los valores más altos corresponden a la tasa de rotación también más elevada (200 rpm), mientras que los más bajos se asocian a la menor velocidad de rotación utilizada $(86 \mathrm{rpm})$. Estas mediciones permitieron visualizar el efecto de la inercia del sistema de barras, lo que se corroboró con el cálculo teórico de esta inercia y la verificación de la similitud de los valores medidos con los teóricos calculados. Tras los torques iniciales, las mediciones muestran que el sistema de barras genera un torque intrínseco entre 1 y $4 \mathrm{Nm}$.

Junto a esta prueba inicial, en taller se efectuó otra prueba que consistió en la excavación de una calicata de $1.5 \mathrm{~m}$ de profundidad y de superficie de $1 \mathrm{~m}$ x $1 \mathrm{~m}$, para luego ser rellenada con arena normalizada suelta y saturada. Se efectuó el ensayo de veleta cuyos resultados se presentan en las Figuras 12 y 13. Las pruebas denominadas Calibración corresponden a los ensayos sin veleta, tal como se explicó en el acápite de metodología de ensayo.

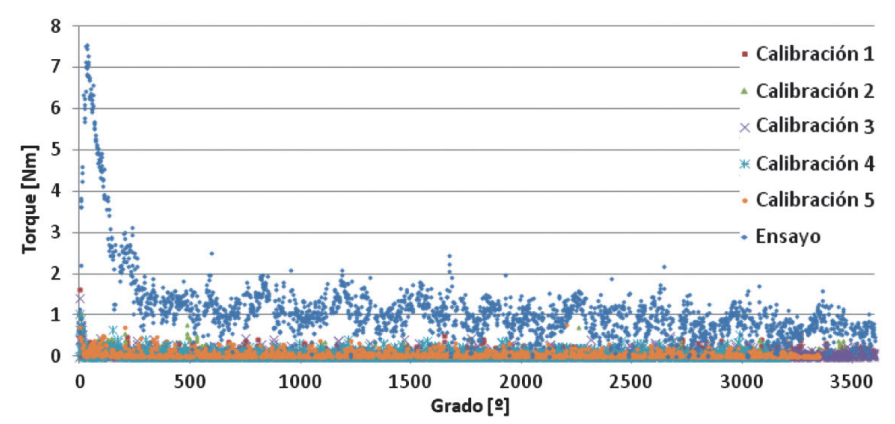

Figura 12: Ensayo de prueba en arena suelta saturada.

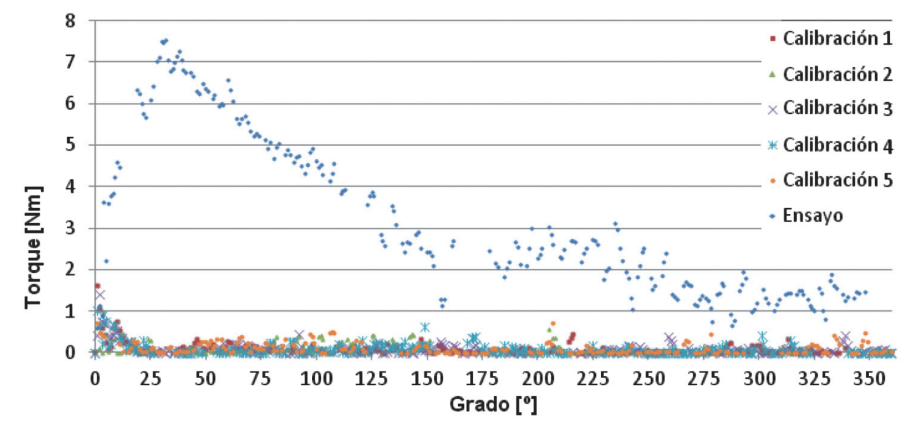

Figura 13: Ensayo de prueba en arena suelta saturada, detallada a $360^{\circ}$

Las Figuras 12 y 13 permiten distinguir claramente la curva torque-ángulo de giro del suelo de las curvas de calibración efectuadas. Así, se obtienen mediciones de torque máximo 
sin corregir en torno a los $7 \mathrm{Nm}$ y torque residual sin corregir en torno a $2 \mathrm{Nm}$ para las arenas saturadas.

\section{Curvas torque - ángulo en lamas de relave}

Las Figuras 14 y 15 muestran dos ensayos de veleta de corte realizados en lamas de relave, a distintas profundidades, a fin de presentar las curvas torque-ángulo de giro que el equipo implementado permite obtener como resultado final.

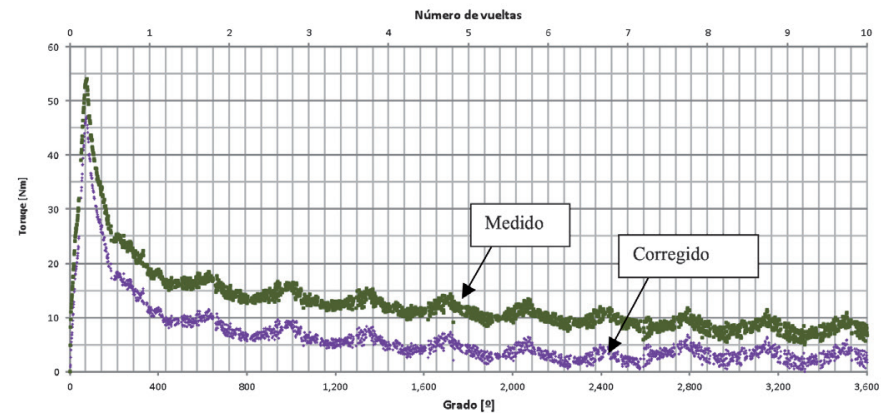

Figura 14: Curva 1 Torque - ángulo de giro en lama de relave. Velocidad de rotación $90 \mathrm{rpm}$

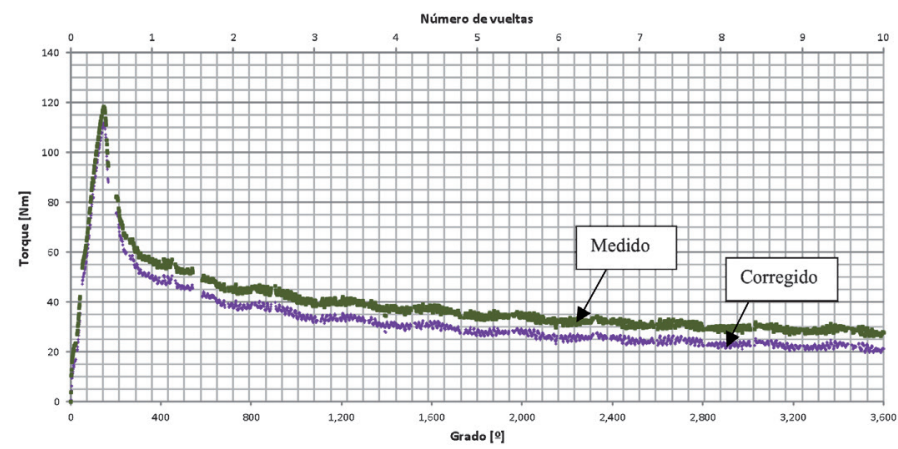

Figura 15: Curva 2 torque - ángulo de giro en lama de relave. Velocidad de rotación $110 \mathrm{rpm}$

De los resultados presentados, cabe señalar el hecho que los torques medidos muestran una tendencia a estabilizarse tras 5 giros -y aun así no se estabilicen por completo durante los ensayos realizados-, sugiere un fenómeno complejo de deformación por corte y de aumento y disipación de la presión de poros en torno a la falla durante el giro de la veleta. En este sentido, notar que en ambos ensayos, tras una vuelta completa de la veleta, la medición del torque viene en descenso. Luego, definir a qué número de vueltas se ha alcanzado el estado residual del suelo pareciera complejizarse, no obstante es evidente considerar que a las diez vueltas de rotación el suelo ya se encuentra completamente remoldeado. A partir de lo anterior, resulta conveniente mencionar la necesidad de futuros estudios orientados a comprender más exhaustivamente el fenómeno físico que se genera sobre la superficie de falla y su entorno al estar sometida a giros con velocidades del orden de las utilizadas en los ensayos presentados, en particular en relación a la transmisión de esfuerzos de corte y aumentos y disipaciones de presiones de poro en el volumen de suelo que se solicita con el giro de la veleta.

\section{Comentarios finales}

La conformación del equipo (implementos mecánicos, eléctricos y sistema de toma y proceso de datos), junto a la metodología empleada para la realización de ensayos de veleta de corte a alta revolución -velocidades angulares en torno a $100 \mathrm{rpm}$ - permite obtener curvas torque-ángulo de giro representativas del comportamiento no drenado de los suelos ensayados, a través de las cuales es posible estimar valores de resistencias al corte no drenadas $S_{u}$, máxima y residuales. En consecuencia, este equipo se constituye como una herramienta adicional a las existentes (tales como CPT-u, ensayos triaxiales, SPT en sondajes) para uso de ingenieros geotécnicos con el propósito de definir la resistencia al corte no drenada de lamas de relave $u$ otros materiales no cohesivos, teniendo en consideración las correspondientes restricciones granulométricas de tamaños máximos, la permeabilidad del suelo y de interpretación de las curvas obtenidas, en especial sobre la resistencia residual en vista de la discusión planteada.

Finalmente, es importante mencionar que durante el proceso de confección del equipo se constató que los principales factores que inciden en una adecuada ejecución del ensayo y obtención de datos son: minimización de giros excéntricos del sistema de barras BQ y veleta de corte a través de uso de barras totalmente verticales (sin curvaturas) y colocación de rodamientos; cuantificación de los torques inherentes al sistema mediante la ejecución de pruebas de calibración o "seteo" antes de efectuar cada ensayo de veleta; reducción de torques parásitos mediante la inclusión de rodamientos; e inserción de la veleta en el suelo mediante un sistema de presión continua, sin inducir ningún golpe o rotación. 


\section{Referencias}

ASTM D 2573 (2008). Standard test method for field vane shear test in cohesive soil. West Coshohocken, PA, USA

Eurocode 7 (2000). Geotechnical Design. Part 3: Design assisted by fieldtesting. DD ENV 1997-3:2000

Ladd, C.C., Foott, R., Ishihara, K., Schlosser, F. and Poulos, H.G. (1977). Stress-deformations and strenght characteristics. State of the art report, IX International Conference of Soil Mechanics and Foundation Engineering, Tokyo, 2, 421-497

Powrie, W. (2004). Soil Mechanics. Concepts and applications. Spon Press, Taylor \& Francis

Wroth, C.P. (1984). The interpretation of in situ soil tests. Géotechnique 34(4), 449-489 